\title{
Voluntary Pupil Size Change as Control in Eyes Only Interaction
}

\author{
Inger Ekman ${ }^{1}$, Antti Poikola $^{1}$, Meeri Mäkäräinen ${ }^{1}$, Tapio Takala ${ }^{1}$ and Perttu Hämäläinen ${ }^{2}$ \\ ${ }^{1}$ Telecommunications Software and Multimedia Laboratory, Helsinki University of Technology \\ P.O.Box 5400, FIN-02015 HUT \\ \{inger.ekman; antti.poikola; meeri.makarainen; tapio.takala\}@tml.hut.fi \\ ${ }^{2}$ Virtual Air Guitar Company \\ Tietäjäntie 4, FIN-02130 ESPOO \\ perttu@virtualairguitar.com
}

\begin{abstract}
We investigate consciously controlled pupil size as an input modality. Pupil size is affected by various processes, e.g., physical activation, strong emotional experiences and cognitive effort. Our hypothesis is that given continuous feedback, users can learn to control pupil size via physical and psychological self-regulation. We test it by measuring the magnitude of self evoked pupil size changes following seven different instructions, while providing real time graphical feedback on pupil size. Results show that some types of voluntary effort affect pupil size on a statistically significant level. A second controlled experiment confirms that subjects can produce pupil dilation and constriction on demand during paced tasks. Applications and limitations to using voluntary pupil size manipulation as an input modality are discussed.
\end{abstract}

\section{ACM Classification Keywords}

H.5.2 User Interfaces: Input devices and strategies, Interaction styles

\section{Keywords}

Pupil size, affective computing, input modality, eye tracking, unconventional computer human interfaces

\section{Introduction}

Pupil diameter is at any time determined by a balance between the competing forces of two smooth muscle systems, controlled by the parasympathetic and sympathetic nervous system, respectively. [Wilhelm et al. 2001] The pupil is constricted by parasympathetic nervous system (PNS) activity which is responsible for the light reflex and near response. Pupil dilation is effected by the sympathetic nervous system (SNS). [Wilhelm et al. 2001.] Pupil size is known to reflect physiological arousal (defined as SNS activation, e.g. [Grings and Dawson 1978]). Arousal is, together with valence, considered one of the primary dimensions of emotional experience and will increase with the intensity of emotional experience. Physiological arousal is, however, not restricted to the emotional experience but is affected by several other processes as well, notably physical activity and cognitive effort.
Use of pupil size has been suggested in affective HCI systems [Janisse 1974; Picard 1997]. However, to date only a few studies have been performed investigating pupil size variations as a source of affective information [Iqbal 2004; Lin and Imamiya 2006; Partala and Surakka 2003; Wang et al. 2006]. In all cases, pupil size has been considered a passive information channel, beyond the users' voluntary control. Our study is also novel in that we provide the subjects real-time information of their pupil size, so that they have constant feedback concerning pupillary behavior.

In this study, we investigate the feasibility of voluntary manipulation of pupil size as an interaction modality. We continue a line of research on unconventional human-computer interfaces, such as using brainwaves, skin conductivity (SC) and heart rate (HR) for controlling a computer. While these methods may at first seem impractical, they nevertheless have their role in the future user interface. For example, brainwaves have been used successfully to control a computer game [Hjelm and Browall 2001]. Pupil based interaction does not try to replace established eye-based interaction techniques (such as dwell time), but broadens the spectrum.

We start by reviewing the literature on pupil size variations in order to identify the factors affecting pupil size and the expected magnitude of pupil size variation. We then proceed to experimentally establish the range of voluntary manipulation by various methods. Following that, we examine pupil size manipulation as an active control method and investigate features crucial to its employment in interaction design. We conclude with a discussion of the practical application of pupil based control.

\section{Previous Research on Pupillary Behavior}

One of the earliest researchers to examine pupil size variations was Bumke, who, in 1911 (as cited in [Hess 1972]), stated that intellectual processes, psychical effort, active mental images, and all affects produce pupil dilation. Bumke also suggested that pupil size can be indirectly manipulated by changing gaze focus from near to far, holding the breath for several seconds, performing muscular effort, or inflicting pain on oneself.

\subsection{Magnitude of Pupil Size Variation}

Pupils will contract several millimeters in response to bright light. Hess [1972] suggested that negative emotions contract the pupils, but the majority of research shows the contrary-both positive and negative arousing stimuli produce pupil dilation [Janisse 1974; Partala and Surakka 2003].

The pupil has been shown to dilate by $10-20 \%$ due to cognitive effort, such as solving multiplication problems [Hess 1972] or translating difficult words from one language to another [Hyönä et 
al. 1995]. The magnitude of dilation is proportional to the difficulty of the task [Kahneman and Beatty 1966].

Studies disagree on the range of emotionally induced pupil variations. Hess [1972] reported $42 \%$ dilation in response to emotionally positive visual stimuli, with only $2 \%$ variation for neutral stimuli. Partala and Surakka [2003] observed on average $0.2 \mathrm{~mm}$ dilation (a few percent) for emotional and $0.15 \mathrm{~mm}$ for neutral stimuli.

\subsection{Speed of Change in Pupil Size}

Hess [1972] reports that emotional stimuli will show a reaction within $2-7 \mathrm{~s}$ from the onset of the stimulus, but suggests that strong emotional stimuli may cause faster reactions. Partala and Surakka [2003] confirm this timeline showing a $0.4 \mathrm{~s}$ delay before pupillary dilation starts, followed by a sudden increase peaking around $2-3 \mathrm{~s}$.

When pupil size is increased due to cognitive processes, changes in mental load are reflected in pupil size, the pupil dilating more for harder tasks and constricting gradually as the task is being completed [Kahneman and Beatty 1966; Hess 1972]. Bernhardt et al. [1996] found that pupil size will drop instantly as soon as cognitive task processing ends.

\section{Design of experiment}

We investigate the extent of control people have on their pupil size. Our experiment is divided into two parts. First, we examine different activities that can affect pupil size and measure how big an impact these activities will have on measured pupil size. The second part involves controlling pupil size on demand in a paced task. The whole test procedure (parts 1 and 2 ) took approximately $60 \mathrm{~min}$. Subjects received movie tickets for participating.

Part 1-Activity categories. Subjects are instructed to try and affect pupil size as much as they can by performing a set of actions provided within each action category. After getting familiar with the eye tracking setup, subjects were introduced to the task and given instructions on manipulating their pupil size. Seven sets of instructed activities (detailed later) were tested repeating the following procedure: Each method is explained, and the subject is allowed a free trial period. Pupil baseline is recorded, after which the subject will actively try and produce extreme high and low values (in this order) using the given set of activities. The subjects decide themselves when they are ready and feel they cannot produce further extremes. Finally, the subjects fill in questionnaires concerning the subjective experience of using the method for controlling pupil size.

Part 2-Paced tasks. Subjects are encouraged to freely form a best strategy for pupillary control using any set of methods tried in part 1 of the test and allowed a short period of free time to try out methods, practice, and recalibrate the system while they do so. After subjects are comfortable with their method(s) of choice, they are asked to produce a series of five alternating highs and lows (in this order) during time slots of ten seconds each, with no pause in between. Total task time 100s consists of 5 'high' and 5 'low' trials $10 \mathrm{~s}$ each. After this, subjects fill in a similar questionnaire as in part 1. Additionally, subjects are asked to describe in their own words what methods or approaches they used to affect pupil size during 'high' and 'low' tasks.

\subsection{Measuring Pupil Size and Providing Feedback}

Seven subjects ( 2 females and 5 males) with normal or correctedto-normal vision participated in the tests. The average age was 33.4 , ranging from 28 to 51 . One subject had prior experience of gaze-based interfaces (from an earlier laboratory visit).
Tests were carried out on a Tobii 1750 eye tracking device. The system measures eye positions and pupil sizes at $50 \mathrm{~Hz}$. [Tobii 2005]. To eliminate system noise, pupil size values were median filtered with a 29 sample window. Outlier values (e.g. pupil sizes of 0 during blinks) are marked and handled as missing data.

We monitored pupil size relative to an individually determined baseline pupil size. Baseline calibration against an empty background was repeated in the beginning of each test task. During the individual tasks, subjects constantly received feedback on the pupil size relative to the baseline in the form of a non-filled black circle on a neutral green background. During the tasks, achieved high or low values were drawn on the screen as red circles. Circles were selected as feedback since they are more intuitive to interpret than numerical values. During tasks, desired direction was indicated with a small arrow in the middle of the pupil feedback circle pointing either outwards or inwards. Feedback also made it easier for subjects to keep their gaze stationary in the middle of the screen.

\section{Activity Categories}

Activities with known effects on pupil size were grouped into seven categories, detailed in Table 1 . The activities consist of both physical and mental tasks. In addition to specific methods, one condition also gave nonspecific instruction related directly to monitoring and modifying the visual pupil feedback: subjects were asked to use concentration and willpower to manipulate the size of the pupil feedback circle.

Table 1. Activity categories listed with instructed methods for controlling pupil size.

\begin{tabular}{|c|c|}
\hline Category & Instructed methods of pupil control \\
\hline Physical activity & $\begin{array}{l}\text { Muscular effort and physical activities such as } \\
\text { holding one's breath. }\end{array}$ \\
\hline $\begin{array}{l}\text { Self-induced } \\
\text { pain }\end{array}$ & $\begin{array}{l}\text { Various (mild) forms of self-induced pain, } \\
\text { pain-related thoughts. }\end{array}$ \\
\hline $\begin{array}{l}\text { Positive } \\
\text { emotions }\end{array}$ & $\begin{array}{l}\text { Positive thoughts and emotion-related behavior } \\
\text { (smiling, laughing). }\end{array}$ \\
\hline $\begin{array}{l}\text { Negative } \\
\text { emotions }\end{array}$ & $\begin{array}{l}\text { Negative thoughts and emotion-related } \\
\text { behavior (frowning, clenching teeth). }\end{array}$ \\
\hline Cognitive tasks & $\begin{array}{l}\text { Performing calculations and reversing words in } \\
\text { their mind. }\end{array}$ \\
\hline $\begin{array}{l}\text { Point of } \\
\text { focus }\end{array}$ & $\begin{array}{l}\text { Focusing the gaze on a point near or in the } \\
\text { distance. }\end{array}$ \\
\hline Concentration & $\begin{array}{l}\text { Concentrating on making the feedback circle } \\
\text { grow or shrink. }\end{array}$ \\
\hline
\end{tabular}

The instructed activities are not assumed to be mutually exclusive, and using one method sometimes includes partial use of another. Tight control of subject behavior was not considered necessary for our study purpose. Since our motive is to investigate whether some methods are useful for consciously and voluntarily modifying pupil size, a failure on the subjects' part to perform according to instructions is in itself a sign that the method is less useful as an interaction technique.

\section{Results}

Achieved pupil control varied greatly between subjects, but both tests support our hypothesis of voluntary pupil control. 
Part 1. Achieved highs and lows for seven methods vary between subjects. Average highs and lows for each method, and standard deviations, are shown in Table 2. Comparisons for statistic significance are made against data measured during inactivity in the beginning of the whole test session, before subjects had received any information on activities affecting pupil size. Repeated measures ANOVA on the range of methods against control values show that some, but not all, methods are distinguishable from the natural variation during inactivity. Methods showing a statistically significant increase in range are indicated by the asterisks in the last column of Table 2 .

Table 2. Achieved LOW and HIGH for seven instructed methods of voluntary pupil size manipulation. LOW and HIGH values are averaged between subjects and reflect extreme deviation from baseline. Standard deviations are shown in neighboring columns. An asterisk in the last column indicates statistically significant increase in range. Level for declaring significance is 0.05 .

\begin{tabular}{|l|l|l|l|l|l|}
\hline Category & LOW & stdev & HIGH & stdev & \\
\hline Physical activity & $-18.8 \%$ & 13.2 & $28.2 \%$ & 18.2 & $*$ \\
\hline Self-induced pain & $-14.0 \%$ & 9.4 & $27.0 \%$ & 11.3 & $*$ \\
\hline Positive emotions & $-18.8 \%$ & 15.1 & $19.3 \%$ & 16.2 & $*$ \\
\hline Negative emotions & $-19.5 \%$ & 12.1 & $15.5 \%$ & 8.6 & \\
\hline Cognitive tasks & $-16.1 \%$ & 13.2 & $15.4 \%$ & 11.8 & \\
\hline Point of focus & $-29.6 \%$ & 12.5 & $13.2 \%$ & 11.3 & $*$ \\
\hline Concentration & $-22.2 \%$ & 12.5 & $11.6 \%$ & 11.1 & \\
\hline
\end{tabular}

Previous research indicates that pupil size should stay relatively stable for alert subjects [Wilhelm et al. 2001]. The large variations observed here indicate that subjects are indeed able to voluntarily control their pupil size. However, the individual differences are large and also affected by the positioning of baseline. This suggests that setting general thresholds for determining pupil effort may not be viable, and a better solution would be to monitor individual differences on a time scale.

Table 3. Methods for manipulating pupil size as reported by 7 subjects. Answers compiled from unstructured textual descriptions given by all subjects after the test.

\begin{tabular}{|l|l|l|l|}
\hline \multirow{2}{*}{ Category } & \multirow{2}{*}{ Details } & \multicolumn{2}{|l|}{$\begin{array}{l}\text { Used by nr. of } \\
\text { subjects }\end{array}$} \\
\cline { 3 - 4 } & & HIGH & LOW \\
\hline \multirow{5}{*}{ Physical activity } & tensing shoulders & 2 & \\
\cline { 2 - 4 } & breath (inhale) & 1 & 1 \\
\hline & pain (pinching) & 2 & \\
\cline { 2 - 4 } & relaxation, calm & 1 & 5 \\
\hline & other & 1 & \\
\hline \multirow{5}{*}{ Emotions } & positive thoughts & 4 & \\
\cline { 2 - 4 } & $\begin{array}{l}\text { positive physical activation } \\
\text { (smiling) }\end{array}$ & 1 & \\
\cline { 2 - 4 } & Aggression & 2 & \\
\cline { 2 - 4 } & detach from emotions & & 1 \\
\hline & exciting thoughts & 1 & \\
\hline Cognitive tasks & $\begin{array}{l}\text { clearing of mind, avoiding } \\
\text { thoughts }\end{array}$ & & 1 \\
\hline Point of focus & close focus & & 1 \\
\hline Feedback-related & $\begin{array}{l}\text { Concentrate on making } \\
\text { feedback circle constrict }\end{array}$ & & 5 \\
\hline
\end{tabular}

Part 2. Given the results for different methods in part 1 , the first question is, what methods subjects use to control their pupil, given a free choice? Questionnaires filled out after the task reveal subjects used a variety of methods, listed in Table 3.

The single most popular form of affecting the pupil was thinking positive thoughts. Notably, while some subjects also used physical activation, there were only two subjects who used exclusively physical activation, pain and aggression to dilate the pupils. These were also the only ones not to show reliable difference in pupil size between 'high' and 'low' tasks; for the rest pupil sizes during the two task conditions showed statistically significant difference. The test was performed as a repeated measures t-test for each subject on the median pupil values during each task, level for declaring significance 0.05 .

Self-picked pupil control techniques produced similar variations on pupil size as observed in part 1 of the test. Repeated measures ANOVA on the whole set of methods reveal not only significant difference to the control condition, but show no difference to the strongest methods of the previous task, namely physical activity, self-induced pain and point of focus.

Finally, we must investigate the nature of pupil control as a function of time. Whereas our results have confirmed that active manipulation can increase the range of pupillary variation, the temporal nature of change will set limits for how pupil size can be utilised in the interface design. Figure 1 demonstrates the development of pupil size during 'high' and 'low' tasks. Data points for each time position interval are averaged over all trials and subjects. Standard deviation is plotted above and below the averages in dotted lines.

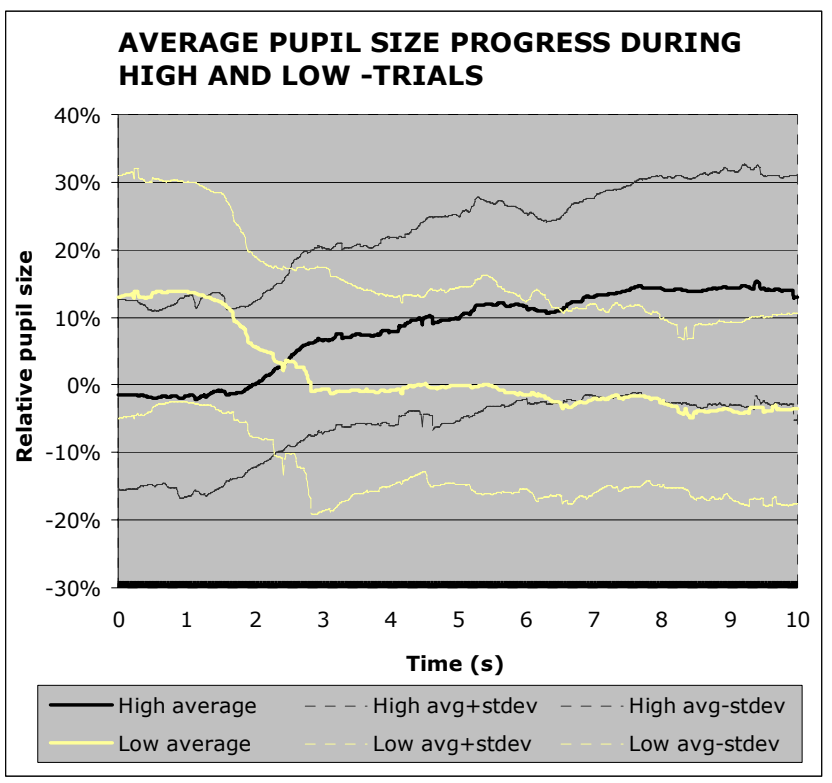

Figure 1. Pupil development during 'high' and 'low' trials. Data points averaged across all trials and subjects. Averages shown in bold with dark and light lines representing 'high' and 'low' trials, respectively. Standard deviations in dotted lines above and below the averages.

There is a clear trend in pupil behaviour. Pupil size starts to constrict and dilate systematically after the change in task objective. The pupil will then slowly reach its ultimate level during the final seconds of each task trial. The time to reach peak value is in line with previous research on stimulus based reactions, indicating that voluntary manipulation operates on a similar time scale. The pupillary behaviour during the early 
beginning of each task should be considered with care, as part of the trend is explainable by the lack of pause between trials. It is probable that voluntary pupil changes take more time to begin than do spontaneous reactions. On the other hand, some changes in voluntary manipulation may also be immediately reflected in pupil size, as pupil size drops in response to task conclusion.

\section{Conclusion and future work}

This study complements extant research on eyes only interaction with an empirical investigation on the voluntary control of pupil size changes. We have shown that when coupled with real time feedback, one can learn to exert control on their pupil size. Seven specific activities were investigated as well as a free combination of methods during paced tasks. The results show statistically significant increases in pupillary size variation for physical activity, self-induced pain, positive emotions and the changing of gaze focus point between near and far points as well as for the free combination of methods determined by the subjects themselves. Pupil behaviour during paced tasks indicates that pupil size can be repeatedly manipulated on demand. Based on these results, we propose that voluntary pupil size is viable for use as an input modality.

Combining pupil size with previous methods within gaze-based interaction increases the number of controls available for eyesonly interaction. Pupil size could also be used as an additional input modality alongside other interaction forms. One compelling reason to use eye tracking in favor of other methods is that a camera can provide these measures wirelessly and allowing hands free interaction.

Whereas pupil manipulation is possible with bodily activation methods (such as muscle effort and self inflicted pain), most interesting aspect for future research in our view is that pupils provide a communication channel for expression of emotional activity. When given free choice of methods, most subjects spontaneously chose positive thoughts as one component of their pupil control and, furthermore, used these methods successfully to manipulate their pupil size. This specific aspect is significant in utilizing pupil manipulation in the interface.

Having a modality controlled mainly by emotional self-regulation opens new avenues for research in affective interfaces. While the nature of the pupil control sets some limitations to the kind of interaction that is feasible, there are several application types where active pupil manipulation could be utilized. Especially, as an active input, pupil size is providing a new way of modelling emotional challenge in social settings. We propose using pupil size as a modifier in affective interpersonal communication channels and social applications. Pupil size can also model mental skills and thus simulate a range of new game mechanics involving social skills, such as seduction and negotiation or abilities calling for extreme levels of concentration like magic. Such game mechanics are presently very hard to realize for the primarily motor interface tools available. For example, the intensity of a magic spell in a fantasy role playing game could be boosted by concentrating hard to dilate one's pupils while a successful lie would require looking a character in the eye for a given time while simultaneously maintaining normal pupil variation.

Before pupil based control can be fully utilized, further research is necessary to understand the design implications and limitations of this new type of control. Compared to the literature, the range of normal pupil variation found in this study was surprisingly high. More thorough investigations are thus needed to establish the normal variation range of pupil size during the use of interactive systems. Also, effort should be made to investigate alternative methods for forming a baseline. Future research should also address how the type and level of detail of the feedback channel influences pupil control. Interesting is also to investigate the transferability of control to a setting without feedback and whether the level of control is increased with training.

\section{References}

Bernhardt P. C., DABbs J. M. ANd Riad J. K. 1996. Pupillometry System for Use in Social Psychology. Behavior Research Methods, Instruments, \& Computers, 28, 61-66.

Grings, W.W. \& DAwson, E. 1978. Emotions and Bodily Responses: A Psychophysiological Approach. NY: Academic Press.

Hess E. H. 1972. Pupillometrics. In Greenfield N. S. and Sternbach R. A. eds. Handbook of Psychophysiology. Holt, Rinehart \& Winston, 491-531.

HJelm S. I. AND Browall, C. 2003. Research + design: the making of Brainball. Interactions, 10 (1), 26-34.

HyönÄ J., TOMmola J. AND AlajA A. M. 1995. Pupil dilation as a measure of processing load in simultaneous interpretation and other language tasks. Q. J. Exp. Psychol. A, 48, (3), 598-612.

IQBAL S. T., Zheng X. S. AND BAILEy B. P. 2004. Task-evoked pupillary response to mental workload in human-computer interaction. CHI '04 extended abstracts. ACM Press, 14771480 .

JACOB, R.J.K. 1996. The future of input devices. ACM Comput. Surv., 28 (4), 138.

JANisse M. P. 1974. Pupil size, affect and exposure frequency. Social Behavior and Personality, 2 (2), 125-146

Kahneman D. And Beatty J. 1966. Pupil Diameter and Load on Memory. Science, 154, 3756, 1583-1585.

LIN T. AND IMAMIYA A. 2006. Evaluating usability based on multimodal information: an empirical study. Proceedings of the 8th international conference on Multimodal interfaces. ACM Press, 364-371.

Partala T. AND SurakKa V. 2003. Pupil size variation as an indication of affective processing. International Journal of Human-Computer Studies. Vol. 59, (1) 185-198.

PICARD R. W. 1997. Affective computing. MIT Press, Cambridge, MA, USA.

Tobir Technology AB. 2005. User Manual Tobii Eye Tracker and ClearView analysis software.

Wang H., Chignell M. AND IshizUKA M. 2006. Empathic tutoring software agents using real-time eye tracking. Proceedings of the 2006 symposium on Eye tracking research \& applications. ACM Press, 73-78.

Wilhelm, B. Giedke, H. LuÈdtke, H, Bittner, E. Hofmann, A. \& Wilhelm, H. 2001. Daytime variations in central nervous system activation measured by a pupillographic sleepiness test. Journal of Sleep Research 10, 1-7. 